\title{
How They Moved and Lifted Heavy Stones to Build the Great Pyramid
}

\author{
Akio Kato \\ Izushi, Shizuoka Pref., Japan \\ Email: akiokato1521@gmail.com
}

How to cite this paper: Kato, A. (2020). How They Moved and Lifted Heavy Stones to Build the Great Pyramid. Archaeological Discovery, 8, 47-62.

https://doi.org/10.4236/ad.2020.81003

Received: October 10, 2019

Accepted: November 16, 2019

Published: November 19, 2019

Copyright $\odot 2020$ by author(s) and Scientific Research Publishing Inc. This work is licensed under the Creative Commons Attribution International License (CC BY 4.0).

http://creativecommons.org/licenses/by/4.0/

\begin{abstract}
We present a new feasible theory about how the ancient Egyptians moved and lifted heavy stones and how they built the Great Pyramid of Giza around 2500 $\mathrm{BC}$, from the viewpoint of energy management taking account of the vast quantity of the stones needed for the Pyramid. We give our solutions to the following three mysteries of the Pyramid: 1) How they could overcome the difficulty in making the four straight edges of the Pyramid meet in one point, high up in the sky? 2) Why all of chambers and passages (the King's and the Queen's chambers, the Grand Gallery and other passages), except the Subterranean chamber, are away from the central axis about seven meters eastwards? 3) For what purpose they dug the Subterranean Chamber, thirty meters deep?
\end{abstract}

\section{Keywords}

Great Pyramid, Nucleus, Statics, Energy Efficiency

\section{Introduction}

We present a new feasible theory about how the ancient Egyptians moved and lifted heavy stones and how they built the Great Pyramid of Giza, around 2500 B.C. The first thing we should do when we want to build something big is the "geotechnical investigation" of the construction site. Therefore, our theory starts that: First of all, they examined and confirmed that "the bedrock at the construction site on the Giza Plateau has dimensions big enough to sustain the planned Pyramid" by digging it, which explains why the Subterranean Chamber locates thirty meters deep below the surface (Remark 3.1). Then the construction of the Pyramid began, but this embraced a big famous problem with the precise shape of the Pyramid (Isler, 2001: pp. 204-211): How they could overcome the difficulty in making the four straight edges of the Pyramid converge to the apex, in 
spite of the fact that the right spot of the apex always stayed high in the air during the construction? Our solution in Section 3 is new but simple: They utilized a chimney-like central well made "vertical" by "gravity." And this resulted in the presence of void spine, which explains why all of chambers and passages (the King's and the Queen's chambers, the Grand Gallery and other passages), except the Subterranean chamber, are away from the central axis about seven meters eastwards. We further propose in Section 4 and Section 5, from the viewpoint of energy management, how they could move and raise the vast amount of heavy stones. Our idea of moving stones is to "roll" stones using "ropes" only, and is quite practical because "rolling" is much easier than "dragging with sledge." To raise stones, we propose an example of simple ancient lift which may coincide with what Herodotus described as the "machine made of short wooden lengths".

\section{Holes and the Central Well}

In this article we mainly concern about the nucleus of the Pyramid, the essential part from the statics viewpoint. Consider a stone of cuboid. Then it is quite natural to chamfer it slightly in order to prevent damage to its edges and also to allow the introduction of a lever. If we chamfer it somewhat deeply, we can get an octagonal prism like Figure 1, and such extra labor of carving would be traded for the following advantages:

1) Lightens the stone; for example, suppose in Figure 1 that the length $\overline{A^{\prime} D^{\prime}}=\overline{B^{\prime} C^{\prime}}$ is half of $\overline{A D}=\overline{B C}$, then the stone loses one eighth of its weight, more than $12 \%$.

2) An octagonal prism can be moved easily by rolling, not dragging, and this is quite an energy saving (see Section 4).

Note also that newly-appeared faces (dotted in Figure 1) need no polishing as they do not touch other stones in the arrangement of stones. Therefore, the extra carving can be rough not to consume much energy in careful carving. Practically, cube-shaped stones would be chamfered into various modified forms of octagonal prism, not so symmetric like Figure 1. It seems in most pyramids the invisible innermost masonry is not so fine compared with the outermost part (Isler, 2001: p. 202, p. 203), and it is even possible to guess that most of the nucleus is made of rough boulders. Nevertheless, we believe that both (unchamfered) cubic stones and chamfered octagonal-like prisms were used in some parts of the nucleus, including some basic lower courses, where the structural stability is exclusively needed. Let us now show that chamfered octagonal-like prisms are indeed quite useful materials for the construction of the nucleus. Observe first that octagonal columns generate holes, for example, four neighboring octagonal columns automatically make a square hole (a hole of rectangular prism) as can be seen in Figure 2, and such a hole is quite strong against external pressure. If we connect this kind of square holes, we can make passages or wells. Another type of wider octagonal hole can be made by eight neighboring octagonal columns and four subsidiary small stones of square prism or cube as in Figure 2, and 
such octagonal hole is also strong due to its arch structure. (Note that the subsidiary stones need not be as high as the surrounding octagonal columns so that they can even be cubes.) Then a vertical well can be made by piling up such square holes and octagonal holes alternately as in Figure 3. There could be various arrangements of stones for a well, other than Figure 3. Even, a well can be made using only cube-shaped stones so that the use of stones of octagonal prism is not crucial in making a well. For our theory the existence of vertical well itself is more important than the shape of its material. We strongly believe that there existed such a well surrounding the central axis of the Great Pyramid from the center of the base to the apex in order to make the shape of Pyramid precise so that the four lateral edges meet in one point, the apex. We will call such a well "the Central Well" assuming its existence.

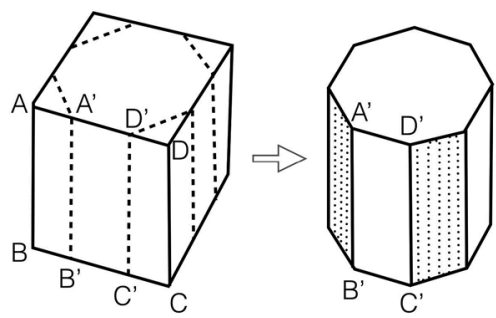

Figure 1. Chamfer a cuboid into an octagonal prism.

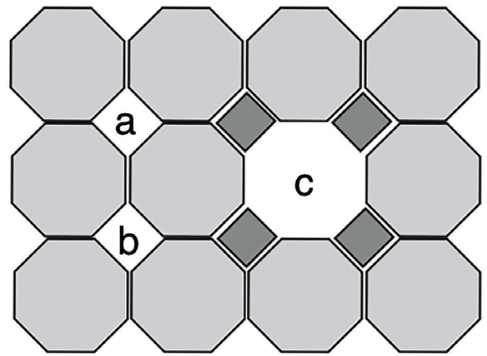

Figure 2. Square holes (a), (b), and octagonal hole (c) (top view).

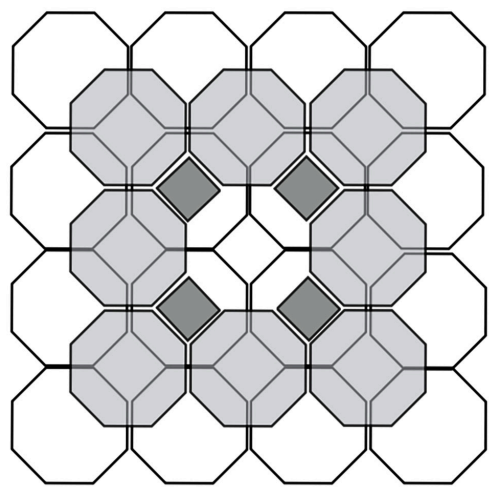

Figure 3. An octagonal hole (made by gray stones) on a square hole (made by white stones) (top view). 


\section{Construction of the Pyramid}

Assumption 1: The whole construction of the Pyramid was led by the vertical well with the central axis.

Let us explain the details of this assumption. Our term "well" stands for an empty column surrounded by a wall of stones so that a "well" need not be underground but rather above the ground like a chimney. Though we have already used the term "vertical well," its precise meaning is that the well has an imaginary axis, vertical to the ground, passing through the centers of all horizontal cross sections of the well (in our case, such cross section is either a square or octagon). First, let us observe that it is always possible to make a vertical well of any height (or depth). Indeed, suppose we have already made a vertical well of some height; then we can increase its height in the following way. Mount some simple framework on the top of the well as shown in Figure 4, and hang a plumb from some point $P$ of the framework down to the base of the well. Adjusting $A, B, C, D$ in Figure 4, find the right spot of $P$ such that the plumb barely touches to the center $O$ of the base of the well as in Figure 5. Then, thanks to the gravity, the plumb line is vertical to the ground and identical to the axis of the well. Guided by this visible axis of plumb line, put new stones of the same height (described as white stones in Figure 5) on the top circumference of the well in such a way that all of them are equidistant from the plumb line. Then we can get a new higher well vertical to the ground, with the axis $P O$ as seen in Figure 5.

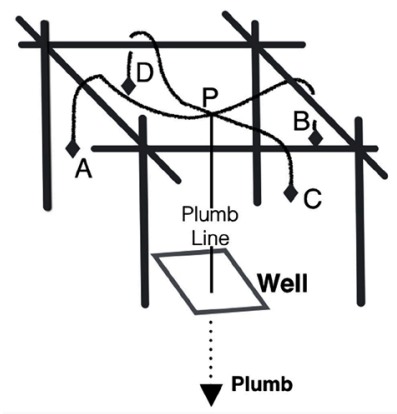

Figure 4. Framework to control the position of the plumb.

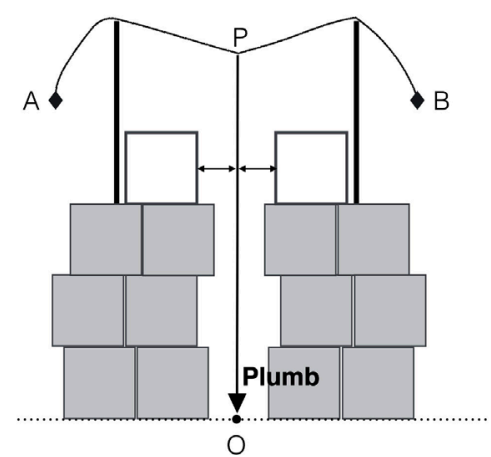

Figure 5. The plumb line $P O$ guides in making a vertical well higher (Vertical section). 
Now, suppose we aim for the final square pyramid $A B C D T$ with the base $A B C D$ and the apex $T$ as in Figure 7. Of course the construction starts with the base $A B C D$. The problem is that the apex $T$ stays always in the sky during the construction, and there is no easy way to identify the exact spot of the apex $T$ to which the four straight edges from $A, B, C, D$ must converge. We can overcome this difficulty by utilizing the vertical well as follows. First, build a vertical well of some height $h$, with its axis $O O^{\prime}$, from the center $O$ of the base $A B C D$ to some point $O^{\prime}$. The height $h$ can be measured precisely as the length of the plumb line $\overline{O O^{\prime}}$; see Figure 6 . Next, around this chimney-like well construct the square frustum $A B C D A^{\prime} B^{\prime} C^{\prime} D^{\prime}$ such that $O^{\prime}$ is the center of the top base $A^{\prime} B^{\prime} C^{\prime} D^{\prime}$ as in Figure 7. Let us show how to find the exact positions of $A^{\prime}, B^{\prime}, C^{\prime}, D^{\prime}$. We already know exact positions of $A, B, C, D, O, O^{\prime}$ as well as the directions $\overrightarrow{O A}, \overrightarrow{O B}, \overrightarrow{O C}, \overrightarrow{O D}$. Let us assume that $\overrightarrow{O A}$ directs to North-East, and the height of the apex $T$ is planned to be $\overline{O T}=146.7$ meters. Then, for example, the desired position of $A^{\prime}$ is at the horizontal distance $\overline{O A} \times\left(\overline{O T}-\overline{O O^{\prime}}\right) / \overline{O T}$ from $O^{\prime}$ toward North-East, and similarly we can find desired positions of $B^{\prime}, C^{\prime}, D^{\prime}$. To find precise orientation it would be helpful to mount a sighting target on the top of the well, e.g., an easy-to-see sign $T^{\prime}$ reflecting the sun as shown in Figure 6 (which would be replaced by the Pyramidion $T$ at the final stage), and no problem in finding precise orientation as the ancient Egyptians were noted astronomers (No problem also in the mathematical ability of the Egyptians in the Old Kingdom, which we can believe in through the fact that the Egyptian in the Middle Kingdom even knew the correct formula for the volume of a square frustum (Gillings, 1972)). Thus, we can obtain the square frustum $A B C D A^{\prime} B^{\prime} C^{\prime} D^{\prime}$ of height $h$ satisfying the strict condition that the extensions of five lines $A A^{\prime}, B B^{\prime}, C C^{\prime}, D D^{\prime}, O O^{\prime}$ meet at the imaginary point $T$ in the sky. Next, make the vertical well heigher $h^{\prime}>h$, with its axis $O O^{\prime \prime}$, and do the similar construction as above to add a new square frustum $A^{\prime} B^{\prime} C^{\prime} D^{\prime} A^{\prime \prime} B^{\prime \prime} C^{\prime \prime} D^{\prime \prime}$ on the old one $A B C D A^{\prime} B^{\prime} C^{\prime} D^{\prime}$. Repeating similar constructions, may be more than ten times, we will be able to pile up frustums to get the final square pyramid $A B C D T$ along with the vertical Central Well OT. Thus we have explained the details of Assumption 1 . To sum up, the whole process resembles how a volcanic eruption creates a pyramidal mountain with the central vent (note that what makes the central vent of a valcano almost vertical is the "gravity" by which the extremely hot gases, lighter than the air, go up straight into the sky from the underground). The Central Well or Vent would have completed its role just when the Pyramidion was set on the top of Pyramid, and its top entrance would have been covered with stones. Therefore, we believe that the Central Well remains as a void column up to some height.

There exists another convincing evidence to support the presence of such spinal cavity. That is the fact that all of chambers and passages (the King's and the Queen's chambers, the Grand Gallery and other passages), except the Subterranean chamber, are away (eastward) from the central axis about seven meters. This is because they needed to avoid the Central Well! 


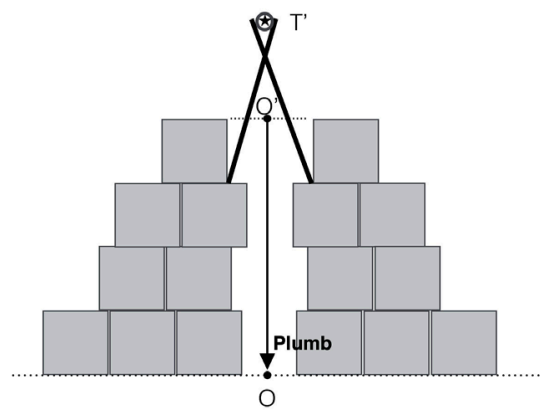

Figure 6. Vertical well mounting a sighting target (Vertical section).

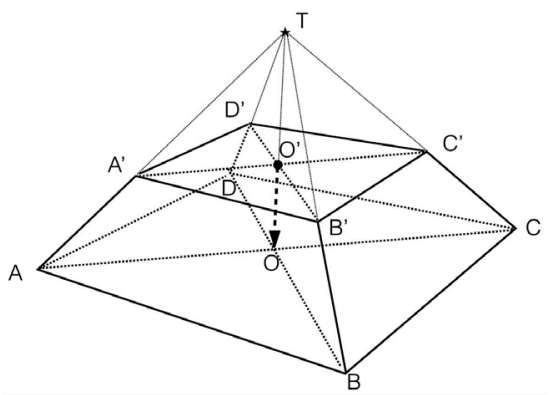

Figure 7. Square frustum with a vertical well $O O^{\prime}$.

Besides the Central Well, we believe there were many vertical wells in order to lift stones (see Section 5) as well as to measure the heights of some tiers. For example, when we want to examine if the plateau $A^{\prime} B^{\prime} C^{\prime} D^{\prime}$ in Figure 7 is horizontal or not, it would be preferable to have vertical wells near the four points $A^{\prime}, B^{\prime}, C^{\prime}, D^{\prime}$ to measure their precise heights. We believe also that square holes were used in setting poles. For example, if we are on the plateau $A^{\prime} B^{\prime} C^{\prime} D^{\prime}$ in Figure 7 and insert poles into all holes there, then we can get an easy-to-see Cartesian coordinate system, which would have worked quite efficiently for supervisors and workmen. Such poles would have helped also in moving and lifting stones as will be explained later in Section 4 and Section 5.

Remark 3.1. Since the Central Well is above the ground, the Subterranean Chamber need not be away from the central axis of the Pyramid. Indeed, it is located just under the center of the base of the Pyramid. One of the mysteries of the Great Pyramid is: For what purpose they dug the Subterranean Chamber, 30 meters deep? We believe the purpose was, as stated in Section 1, the "geotechnical investigation" to examine if the bedrock had the dimensions big enough to sustain the whole Pyramid, which explains also the fact that the Subterranean Chamber looks "left unfinished". They dug two tunnels (see Figure 8) the underground parts of "the Descending Passage" BD and "the Well Shaft" BC, down to the Subterranean Chamber, and then the short tunnel $A B$ toward the south from the Chamber. This digging confirmed them that the bedrock extended at least to the gray part of Figure 8. Since the Giza Plateau appears to extend from north to south and the site in question is the northern corner of the Plateau, we 
believe they carefully checked the northern part of the bedrock by digging $\mathrm{BD}$ and $\mathrm{BC}$, while just $\mathrm{AB}$ for the southern part. Theoretically speaking, the least bedrock needed to sustain the biggest pyramid they were going to build would be the gray part of Figure 9, of the form of upside-down pyramid, 30 meters deep from the center of the base, which is included in that of Figure 8. Thus, this geotechnical investigation gave them the green light for the construction of the Pyramid. And, being supported by such a big enough bedrock, the Great Pyramid has withstood earthquakes until now, for 4500 long years. Remind and compare with the historical fact that the Lighthouse of Alexandria, constructed in the 3rd century $\mathrm{BC}$, was destroyed mainly by earthquakes in the late 10th century and the early 14th century AD, so it could survive about 1500 years, only one third of 4500 years. See (Badawy, 1999) about the earthquakes in Egypt.

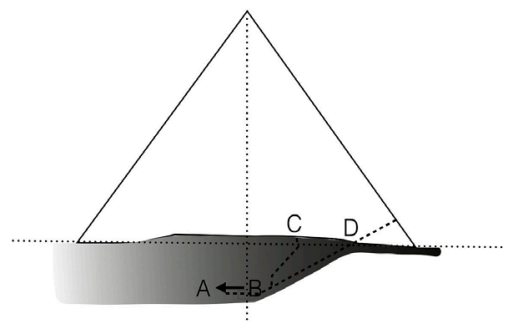

Figure 8. The confirmed dimensions of the bedrock.

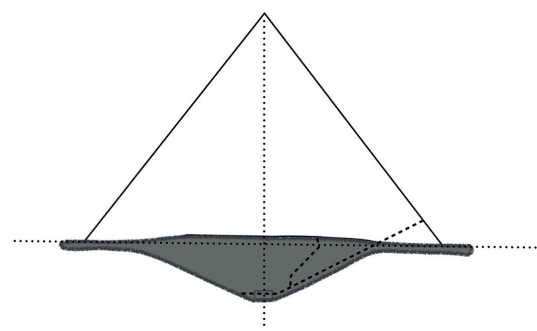

Figure 9. The least bedrock needed to sustain the pyramid.

\section{How to Use Ropes to Move Stones}

We now propose how they moved heavy stones. Note the simple practical fact that, in moving a heavy stone, "rolling" if possible is usually much easier than "dragging." In other words, "rolling friction" is extremely smaller than "sliding friction". We consider two types of stones, a cubic stone and a stone of octagonal prism. Let us first consider the latter. An octagonal prism can be easily moved by "rolling" since it is almost a cylinder. Though, of course, one can push directly or utilize levers to roll such a cylindrical stone, here we show how to use ropes to roll a stone, quite an efficient and simple way with no need of burdensome device like a sledge. Wrap ropes around a stone of octagonal prism as in Figure 10 and pull one end of each rope holding another end. Then the stone will rotate and move. Since the reason to hold one end of each rope is lest the rope slips, 


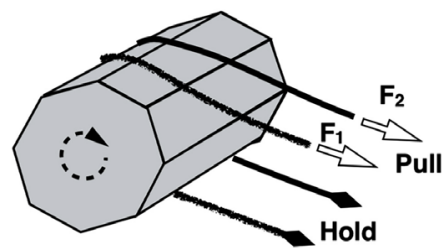

Figure 10. Using ropes to roll a stone.

various alternative ways are possible. For example:

1) Fix one end of each rope to some post (Figure 11);

2) Tie knots in the ropes; if we further join two ends of each rope, we can get a very simple way which looks like a "belt drive" (Figure 12);

3) Lay logs on the ground (Figure 13);

4) Loop each rope around the stone a few times (Figure 14); looping just once is enough if one end of each rope is hold slightly by someone (see also Figure 19);

5) One who pulls a rope stamps on the other end of the rope (this practical way seems to be well known nowadays among Japanese landscape gardeners who need to arrange big natural stones in creating a Japanese Garden) ${ }^{1}$.

General Caution: Most of our figures are simplified to illustrate "force diagram" rather than the actual way. Practically, many strong ropes and thick poles or posts would be necessary.

On a ramp it would be better to use turning posts or well-greased posts as in Figure 15 in order to redirect the pulling force and to utilize our bodies' weight or counterweight effectively. Such a turning post can be considered as a primitive simple pulley; see Section 5. The chocks described in Figure 15 can be stones of triangular prism produced abundantly as a by-product when cubic stones were chamfered.

Remark 4.1. When we pull a stone of octagonal prism $A_{1} A_{2} \cdots A_{8}$ of weight $\mathrm{W}$ using ropes with the horizontal force $\mathrm{F}$, on level ground, the force diagram can be like Figure 16. Let us estimate the size of $F$. The effort $F$ is applied to the point $A_{2}$, and the pivoting point is $A_{6}$. Let $d$ and $h$ be the (perpendicular) distances from the pivoting point $A_{6}$ to the vectors $W$ and $F$, respectively. Then the strength of $F$ needed to rotate the stone is $F=W \cdot d / h$. Let $0 \leq \theta \leq \pi / 8$ be the angle of inclination, i.e., $\theta=\angle A_{5} A_{6} B$, and assume the regularity of the octagon. Then we get

$$
\begin{gathered}
d / h=\frac{1}{2} \tan \left(\frac{\pi}{8}-\theta\right) \\
\left(\approx \frac{1}{2}\left(\frac{\pi}{8}-\theta\right) \text { with the error }<0.011\right),
\end{gathered}
$$

which, starting with the value $0.2071 \cdots \approx 1 / 5 \quad(\theta=0)$, decreases to $0(\theta=\pi / 8)$, and takes the intermediate value $0.0994 \cdots \approx 1 / 10 \quad(\theta=\pi / 16)$. So, the force $F$ necessary to start rolling is almost $W / 5$, and then $W / 10$ at the angle $\theta=\pi / 16$. Almost no power will be needed to keep the stone rolling after the line $A_{2} A_{6}$ stands vertical ( $\theta=\pi / 8$ ), thanks to gravity. Note that when we push the stone, $\underline{\text { https://jflc.or.jp/media/niwa_navi/20120330_1246_33_0706.pdf }}$ 
the effort will be applied to some point about the height $h / 2$ (between $A_{3}$ and $A_{4}$ ) and then we need the power twice the case of pulling. Thus, pulling is better than pushing. Suppose a stone of weight 2.5 tons was chamfered to lose $10 \%$ of its weight, and assume $W=2.5 \times 0.9=2.25$ tons. Then $W / 5=450 \mathrm{~kg}$ which amounts to nine men's power, assuming one man's pulling force is $50 \mathrm{~kg}$. Precisely speaking, we should take account of the energy loss due to the friction between the ropes and the stone. So, practically, in any of Figures 10-14, about ten men will be needed at the beginning (and four ropes provided each rope is pulled by a few men), and about five men at the inclination $\theta=\pi / 16$. Thus, many men are needed at the beginning, but, once the stone starts moving, only a few men would be able to keep it rolling. This is quite an energy saving compared with dragging which needs constant application of power, consuming a lot of energy. We may conclude that, practically, it would be better to be assisted by a lever at the beginning to incline a stone a bit so that only several men can start the roll, and when we suspend rolling the stone, we better use chocks to hold it like Figure 17 which makes easy to resume rolling.

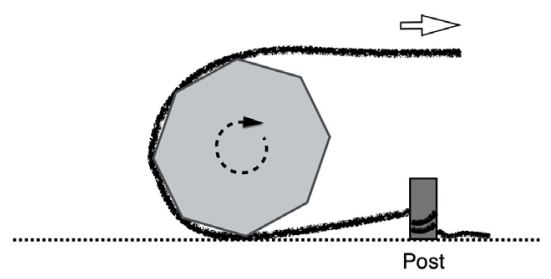

Figure 11. Use posts.

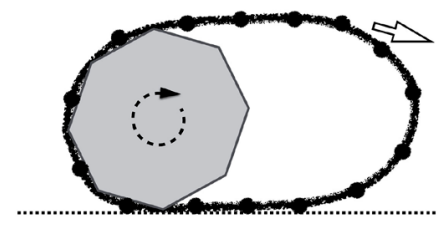

Figure 12. Tie knots.

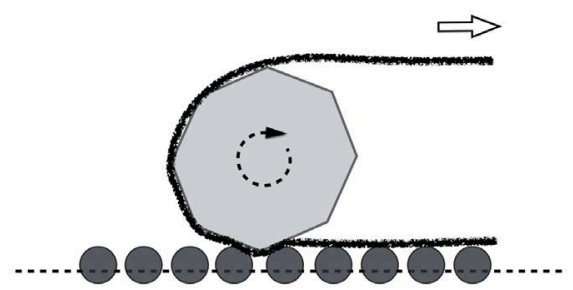

Figure 13. Lay logs.

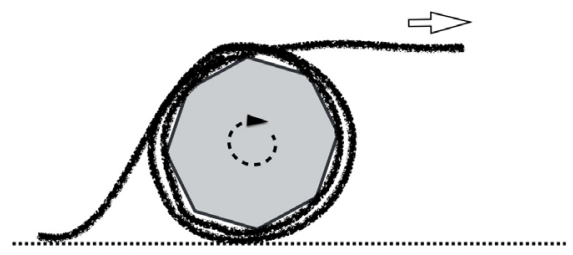

Figure 14. Loop ropes. 


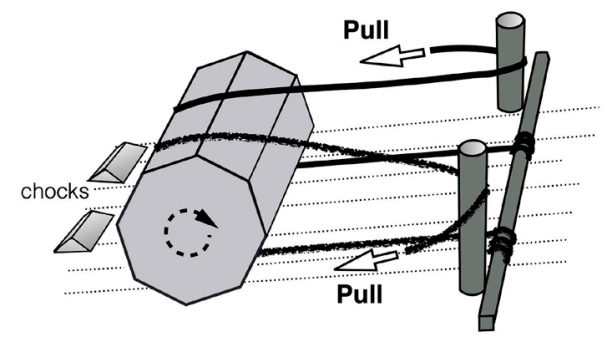

Figure 15. Using turning posts on a ramp.

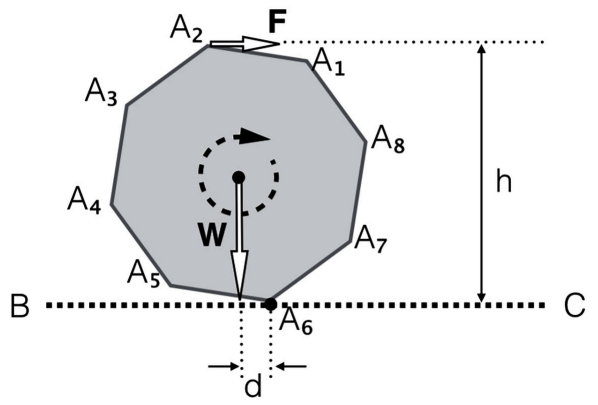

Figure 16. Force diagram when a stone of octagonal prism is pulled by ropes.

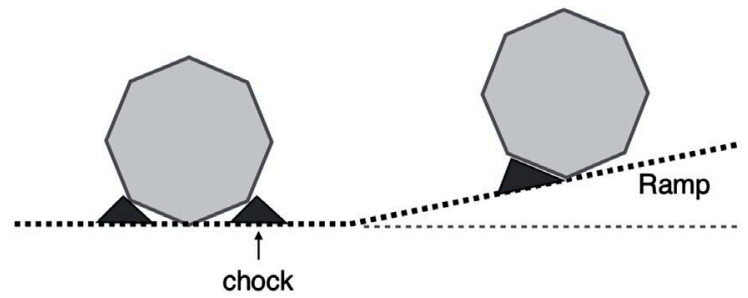

Figure 17. Use of chocks to suspend and resume rolling.

Next, let us consider the case of cube-shaped stone. Of course such a stone might be dragged using a sledge, here we point out that even such a cubic stone can be rolled using ropes only. First of all, note that we can get an octagon by attaching four obtuse triangles to sides of a square. Therefore, if we place stones of triangular prism on the ground like Figure 18 (upside down version of chocks in Figure 15 or Figure 17), rolling a cubic stone is essentially the same as rolling an octagonal prism. Note that such triangular stones can be replaced by any stones of almost the same height as shown in Figure 19, and this simple way could be applied wherever one can find suitable stones. Figure 19 shows also an advantage of using ropes that we can control the speed of rotation, lest the roll damage the stone, by pulling both ends of each rope to adjust the force $F$ - $G$. Instead of stones, logs can be placed on the ground or ramp at regular intervals as in Figure 20, where each interval should be almost the side length of the stone.

Remark 4.2. By chamfering the right edge of a right triangular prism we can get a trapezoidal prism, and such prisms can be used like Figure 21 (similar to Figure 18) which also shows that a stone on such a trapezoidal short prism can be turned easily. One can see in (Fonte, 1998) a kind of semicylinder called "quar- 
ter circle" placed with "the flat side on the ground," which looks like an upside down version of our case of Figure 21. An amazing mathematical fact was discovered by (Robison, 1960) that a square wheel can roll horizontally on a road made up of inverted catenaries as in Figure 22 (where "catenary" is the mathematical name of the curve describing a rope or chain hanging loosely between two supports, and an inverted catenary means the curve upside down). This means that such catenary-abutted road can "completely" suppress the vertical movement of the center of the square. Likewise, in Figure 19 and Figure 21, the laid stones suppress "somewhat" the vertical movement of the center of mass of the cubic stone. We can learn from the Khufu first and second Solar Ships found at the foot of the Pyramid that the ancient Egyptians had outstanding knowledge and skill about ropes. They made ropes from the strong materials (e.g., "flax"), and used long enough ropes to hold the ship's timbers together (Jenkins, 1980). Though being strong against strain, ropes would be somewhat easy to be crushed between a heavy stone and the ground. To avoid such a crush, it will be important to suppress the vertical movement of the center of mass of the stone.

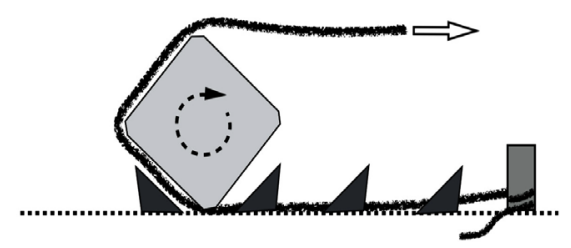

Figure 18. Stones of triangular prism laid regularly on the ground (side view).

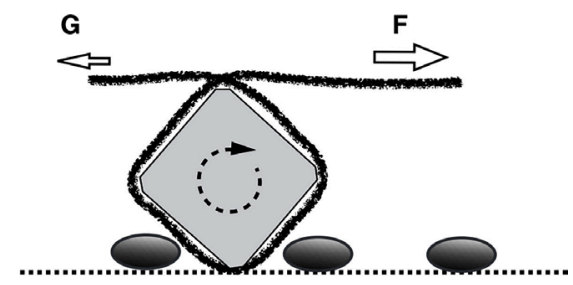

Figure 19. Use of any stones of proper height, and the control of the speed of rotation by the force $F-G$ (side view).

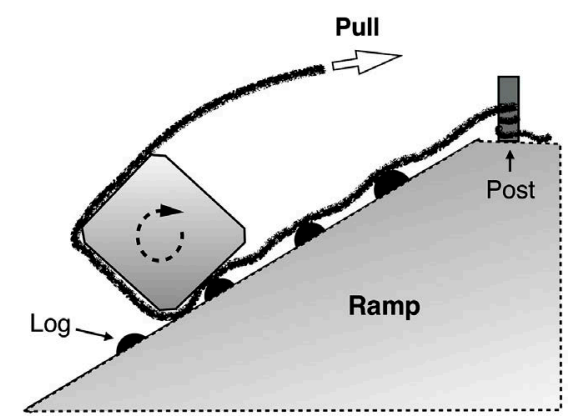

Figure 20. Logs laid regularly on a ramp (side view). 


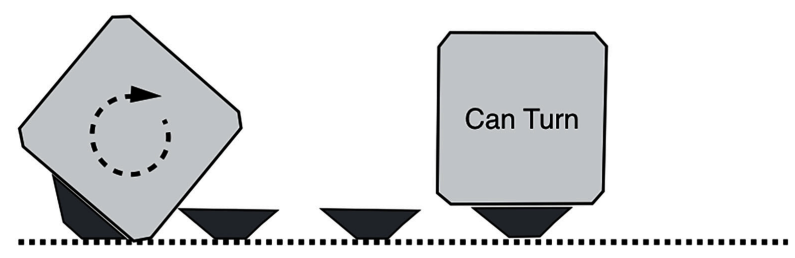

Figure 21. Use of stones of trapezoidal prism.

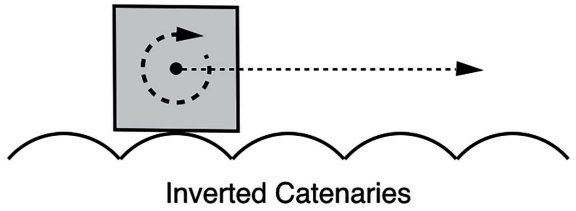

Figure 22. The square can roll on the curve of inverted Catenaries moving its center Horizontally.

\section{Ancient Lift}

We next propose how they lifted stones. From the viewpoint of energy efficiency we assume some low ramps were made to build the lower courses of the nucleus, but large inefficient ramps were not made. So, in order to lift many heavy stones to upper courses, some device was surely needed, and such device we propose is a simple wooden lift illustrated in Figure 23. Here we assume that:

Assumption 2: Forerunners of simple pulley were used with ropes in moving and lifting stones.

This would be quite a reasonable assumption since some devices, believed to be simple pulleys, have been found from the 4th Dynasty on the Giza Plateau, and as noted before, the turning posts in Figure 15 can be considered as "forerunner" of simple pulley (Isler, 2001: p. 262, p. 302). In Figure 23 ropes are fixed at the poles $A, B$ of the platform, and the rotatable poles $C, D$ are "forerunner" of simple pulley. In order to protect poles and ropes from wearing down it would be better if the poles $C$, $D$ were well greased, and much better if some wooden or bronze tubes were inserted between the poles $C, D$ and ropes. Note that the vertical thick posts in this figure can be replaced by any wooden towers as in Figure 24, where two such towers support a rotatable pole. Of course, Figure 23 essentially shows the force diagram, and actually many stronger ropes as well as thicker poles and posts would be necessary. Notice that: the crossed pole $C$ or $D$ turns stronger as the span between the two vertical posts becomes shorter.

Let us estimate the number of men needed to raise a stone using this lift. Noteworthy in lifting is that we can use counterweights effectively as illustrated on the right side of Figure 23 to decrease the number of men in labor. It would be even possible to hold a stone in the air using only counterweights. In particular, we can disregard the weight of the platform of the lift since it is always possible to balance the platform by counterweight. Suppose we are going to lift a stone of weight 2.25 tons (as in Remark 4.1) without any additional counterbalance. One man's "pulling down" force would be much stronger than his hori- 
zontal pulling force $50 \mathrm{~kg}$ assumed in Remark 4.1 since one can use his weight quite effectively by almost hanging on the rope. So, let us assume that one man's pulling down force is about $70 \mathrm{~kg}$ or $80 \mathrm{~kg}$. Then, we may need about 2250/75 = 30 men, and about ten ropes, five on each side, if each rope is pulled by three men. This is quite a number. Though we can decrease this number using counterbalance, in general many men would be needed to lift such a big stone. But this is not the case of smaller stones. For example, a cubic stone of side length half a meter is about $300 \mathrm{~kg}(\approx 2.5 / 8$ tons), and to lift such a stone, we need only four or six men, quite a reasonable number (in case of a stone of side length 0.7 meters, weighing $850 \mathrm{~kg}$, we need about twelve men). The stability of the nucleus would not change at all even if every cubic stone of side length bigger than one meter were replaced by eight stones of half size, and needless to say, smaller stones are much easier to handle with in many respects. Therefore, from the viewpoint of energy management we believe that:

Assumption 3: Smaller stones of side length about a half meter would be used a lot for upper courses of the nucleus.

To raise stone to upper courses, each lift would be set and used like Figure 25 so that a lifted stone would roll onto the upper tier if the platform of the lift was inclined by pulling the ropes on the end. Since the quantity of the stones was vast, we believe various alternative ways were also employed simultaneously to raise stones. One of such ways would be to make vertical wells and set some simple lifts on their tops. An example of such a well with a lift is illustrated in Figure 26, by which smaller stones could be raised efficiently. Though mechanically the same as Figure 26, it would be possible to use two wells like Figure 27. A combination of a stairway and a well would also work as Figure 28 , where the "white" stone can roll up the face of a stairway when the "black" stone, a counterweight, goes down in the well, while the "black" stone can go up in the well when the "white" stone, a counterweight, rolls down the face of a stairway (this looks somewhat like a modern escalator). See (Isler, 2001) about stairways. Vertical wells would be used also to lift other things, e.g., tools, foods, water or even men, like a modern elevator, concluding that vertical wells were quite indispensable from the viewpoint of energy management. And we believe most of such wells would be made on the west side of the Pyramid, since the east side was for important spaces such as the King's chamber, the Queen's chamber, the Grand Gallery and other passages. In short, the west side was a scaffold for the construction of the east side.

Obviously, every well, including the Central Well, needs a tunnel connecting its base to the outside. Such tunnels belong to the lower courses of the nucleus and note that it was possible to set bigger stones in the lower courses using low ramps. So, they could be made wide enough at least in dealing with the smaller stones as in Assumption 3. For the structural stability and for the protection against invaders such tunnels would be filled with stones when they completed their role, and so, they do not remain as empty spaces nowadays, we believe. 

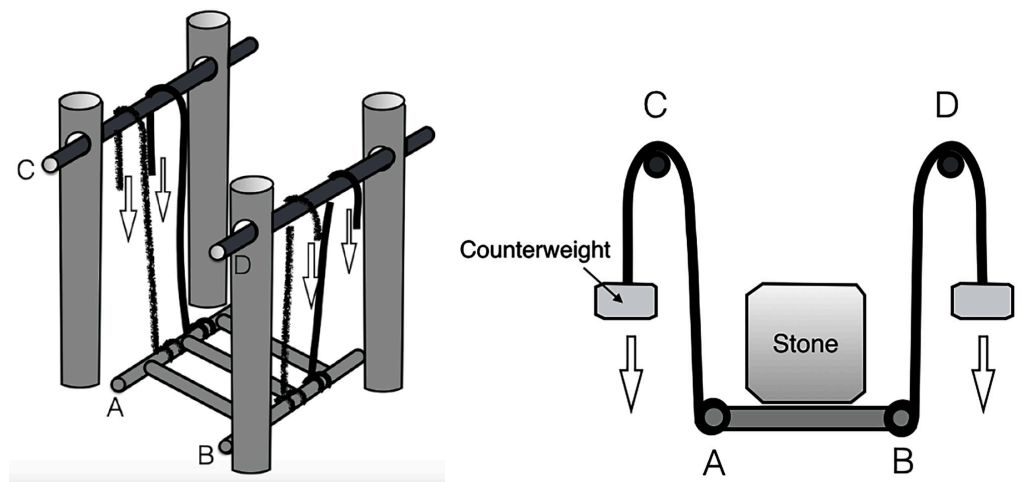

Figure 23. Ancient lift, and its usage.

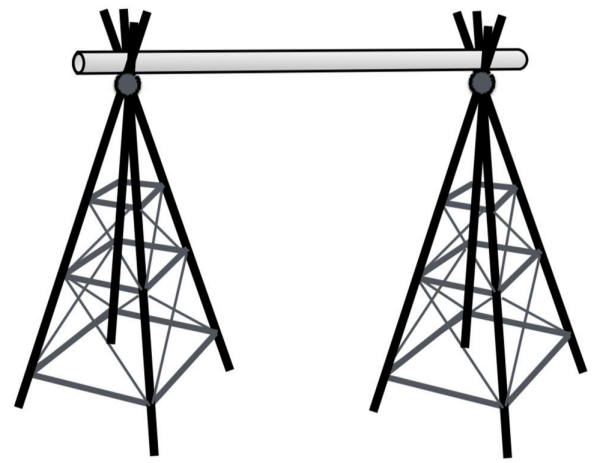

Figure 24. Two wooden towers supporting a rotatable pole.
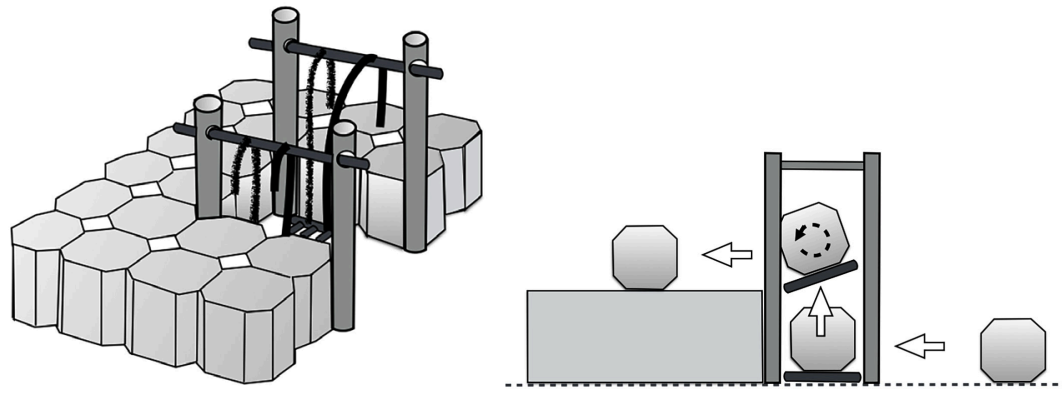

Figure 25. Setting of a lift, and its usage.
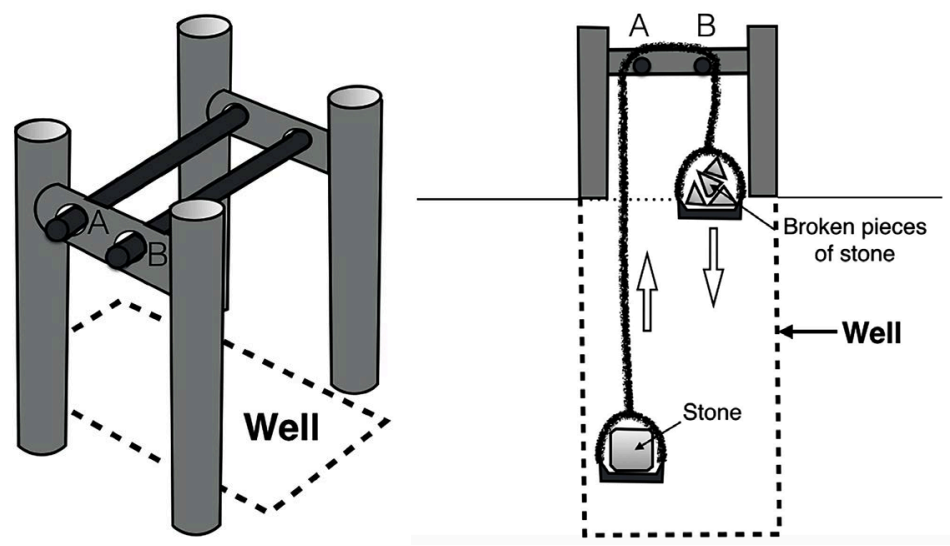

Figure 26. A simple lift on the top of a well, and its usage. 


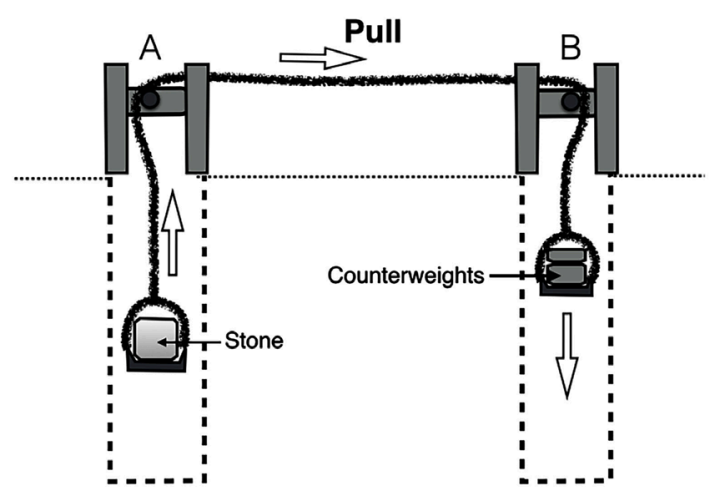

Figure 27. Use of two wells.

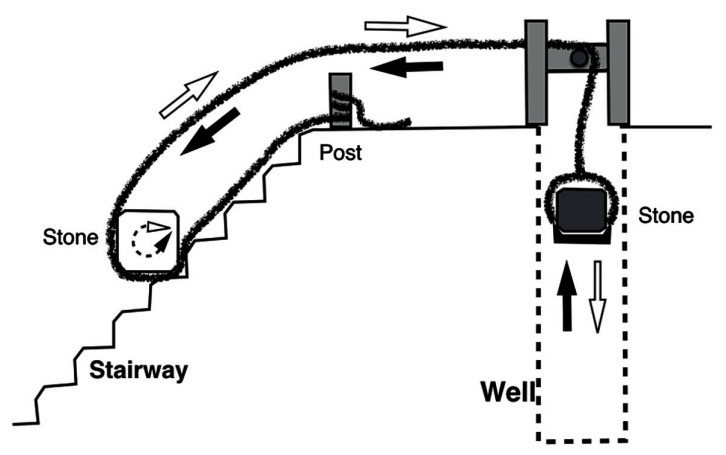

Figure 28. Combination of a stairway and a well.

Remark 5.1. It is feasible that the Ancient Lifts proposed in this section coincide with the "machines made of short wooden lengths" described by Herodotus "The Stories II, 125" (written about 500 BC, i.e., two millennia after the Pyramid Age) as follows:

“... This pyramid was built like this: tier after tier ... they raised the stones ... with machines made of short wooden lengths, lifting the stones from the ground up to the first tier of steps. When a stone had been raised on the first (tier) it was placed above another machine made of short wooden lengths, that was on the first tier, and from this it was lifted to the second tier and placed onto another machine, many were the steps so many were the machines, that (could be) only one, so easy to carry, from tier to tier."

Since the lift of Figure 23 is made of just poles, posts and a platform with ropes, it can be easily taken apart and assembled again, and hence, "so easy to carry, from tier to tier".

\section{Concluding Remarks}

We have presented our idea, from the viewpoints of statics and energy efficiency, about how the ancient Egyptians succeeded in moving and lifting the vast quantity of heavy stones to build the Great Pyramid, setting reasonable Assumptions 1,2 and 3. Let us add the following remarks:

1) Quite recently, in 2018, there was a remarkable discovery, which would 
support our theory about moving stones in Section 4, that is, the finding ${ }^{2}$ of the ancient ramp system at site in Egypt's eastern desert slope. This ramp system is lined with two staircases and wooden posts, into which our Figure 15 and Figure 20 can fit very well.

2) About the Central Well, we now strongly hope to get some direct evidence for the presence of void column for the Central Well, and we wonder if it is possible to confirm such presence, somehow without destroying any parts of the Pyramid, recalling the recent discovery of a big void in the Pyramid (Morishima et al., 2017).

Since the author is a mathematician ${ }^{3}$ working on "Topology", a branch of modern geometry, this article took somewhat a mathematical style setting some "Assumptions" to get some conclusions.

\section{Conflicts of Interest}

The author declares no conflicts of interest regarding the publication of this paper.

\section{References}

Badawy, A. (1999). Historical Seismicity of Egypt. Acta Geodaetica et Geophysica Hungarica, 34, 119-135.

Fonte, G. C. A. (1998). Building the Great Pyramid in a Year. London: Professional Engineering Publishing.

Gillings, R. J. (1972). Mathematics in the Time of the Pharaohs. Cambridge, MA: MIT Press.

Isler, M. (2001). Sticks, Stones, and Shadows Building the Egyptian Pyramids. Norman, OK: University of Oklahoma Press.

Jenkins, N. (1980). The Boat beneath the Pyramid. Holt, New York, NY: Rinehart and Winston.

Morishima, K., Kuno, M., Nishio, A., Kitagawa, N., Manabe, Y., Moto, M. et al. (2017). Discovery of a Big Void in Khufu's Pyramid by Observation of Cosmic-Ray Muons. Nature, 552, 386-390. https://doi.org/10.1038/nature24647

Robison, G. B. (1960). Rockers and Rollers. Mathematics Magazine, 33, 139-144. https://doi.org/10.2307/3029034

${ }^{2}$ https://www.dailymail.co.uk/sciencetech/article-6359375/Ancient-Egyptian-ramp-used-drag-huge-s tone-blocks-uncovered-4-500-year-old-quarry.html

${ }^{3}$ retired from the National Defense Academy, Japan, in 2015. 